\title{
Application of the Evolution-Variable Manifold Approach to Cavity-Stabilized Ethylene Combustion
}

\author{
Niccolo Cymbalist* \\ California Institute of Technology, Pasadena, CA \\ Graham V. Candler ${ }^{\dagger}$ \\ Aerospace Engineering and Mechanics, University of Minnesota, Minneapolis, MN \\ Paul E. Dimotakis ${ }^{\ddagger}$ \\ California Institute of Technology, Pasadena, CA
}

\begin{abstract}
For combustion in high-speed flows, radical-formation time scales and ignition delay times may be similar to, or dominate, relevant flow time scales. Reliable modeling of induction and autoignition processes is critical to the prediction of combustor performance. The evolution-variable manifold (EVM) approach of Cymbalist and Dimotakis ${ }^{1}$ uses a transported scalar to track the evolution of the reaction processes, from induction leading to autoignition and subsequent robust combustion. In the present work, the EVM method is implemented in a computational fluid dynamics code in which wall-modeled large-eddy simulations are performed for two ethylene-air high-speed combustion cases. The detailed thermochemical state of the reacting fluid is tabulated as a function of a reduced number of state variables that include density, energy, mixture fraction, and the reaction-evolution variable. A thermodynamically consistent numerical flux function is developed and the approach for coupling the large-eddy simulation to the EVM framework is discussed. It is found that particular attention must be given to the solution of the energy equation to obtain accurate and computationally stable results. The results show that the LES-EVM approach shows promise for the simulation of turbulent combustion of hydrocarbons in high-speed flows, including those dominated by ignition delay, and encompass regions of thin reaction fronts as well as distributed reaction zones.
\end{abstract}

\section{Nomenclature}

$\begin{array}{ll}a & \text { speed of sound }(\mathrm{m} / \mathrm{s}) \\ A & \text { convective flux vector Jacobian matrix } \\ c_{v}, c_{v s} & \text { mixture and species specific heat }(\mathrm{J} / \mathrm{kg} \mathrm{K}) \\ e, e_{s} & \text { mixture and species energy per mass }(\mathrm{J} / \mathrm{kg}) \\ \mathcal{C}, \mathcal{X}, \mathcal{Z} & \text { progress variable, non-fuel, and fuel mass fractions } \\ D & \text { diffusion coefficient }\left(\mathrm{m}^{2} / \mathrm{s}\right) \\ E & \text { total energy per volume }\left(\mathrm{J} / \mathrm{m}^{3}\right) \\ E_{\text {int }} & \text { internal energy per volume }\left(\mathrm{J} / \mathrm{m}^{3}\right) \\ F & \text { convective flux vector } \\ h, h_{o} & \text { enthalpy and total enthalpy }(\mathrm{J} / \mathrm{kg}) \\ i & \text { grid index } \\ k & \text { kinetic energy }(\mathrm{J} / \mathrm{kg})\end{array}$

*Graduate Research Assistant, Student Member AIAA

${ }^{\dagger}$ Russell J. Penrose and McKnight Presidential Professor, Fellow AIAA

‡Professor of Aeronautics \& Applied Physics, Fellow AIAA 


$\begin{array}{ll}L, R & \text { values obtained from left and right data } \\ M_{s} & \text { molar mass of species } s(\mathrm{~kg} / \mathrm{kmol}) \\ \hat{n}, n_{x}, n_{y}, n_{z} & \text { element face unit normal vector and components } \\ p & \text { pressure }(\mathrm{Pa}) \\ \hat{R} & \text { universal gas constant }(\mathrm{J} / \mathrm{kmol} \mathrm{K}) \\ \bar{R} & \text { mixture gas constant }(\mathrm{J} / \mathrm{kg} \mathrm{K}) \\ R, R^{-1} & \text { eigenvector matrices } \\ s & \text { species in detailed kinetics model } \\ T & \text { temperature }(\mathrm{K}) \\ t & \text { time }(\mathrm{s}) \\ t_{d, c} & \text { characteristic ignition-delay time }(\mathrm{s}) \\ U & \text { vector of conserved variables } \\ \vec{u}, u, v, w & \text { velocity vector and components }(\mathrm{m} / \mathrm{s}) \\ u^{\prime} & \text { face-normal velocity }(\mathrm{m} / \mathrm{s}) \\ V & \text { vector of primitive variables } \\ \dot{\omega}_{s} & \text { chemical source term of species } s\left(\mathrm{~kg} / \mathrm{m}^{3} \mathrm{~s}\right) \\ x_{j} & \text { coordinate directions } \\ Y_{s} & \text { mass fraction } \\ \alpha_{d} & \text { dissipative flux factor } \\ \alpha_{s} & \text { species weights } \\ \Lambda & \text { diagonal matrix of eigenvalues } \\ \lambda & \text { eigenvalue } \\ \rho_{s} \rho_{s} & \text { evensity, species } s \text { density }\left(\mathrm{kg} / \mathrm{m}^{3}\right) \\ \chi & \text { entrainment rate } \\ & \end{array}$

\section{Introduction}

Reliable simulation of turbulent combustion in high-speed air-breathing propulsion is important for engine design and optimization. The most promising approach for representing these complex flows is large-eddy simulation (LES) modeling, in which large-scale turbulent motions are resolved and small-scale effects are modeled. There are several widely used approaches for the LES of turbulent combustion, but most were originally developed and applied to the study of combustion occuring in low-speed flows. In high-speed flows, important additional effects include compressibility, energy coupling, and ignition delay. During induction, and prior to ignition, convected fuel elements undergo slow chemical reactions until a critical level of radicals is produced, followed by ignition, rapid oxidation, and heat release. Because of the high-speed conditions, this induction or ignition delay may take place over a very large physical distance in the combustor, and can dominate the overall engine performance.

Cymbalist and Dimotakis ${ }^{1}$ have shown that detailed chemical kinetics models for ethylene exhibit large variation (up to an order of magnitude) in predicted ignition-delay time at relevant conditions in air. They propose a hybrid model in which induction and subsequent ignition and heat-release processes are represented by a transported evolution (progress) variable, whose evolution rate is derived from shock tube data prior to ignition, and detailed chemical kinetics post ignition. Experimental shock-tube data are used to build a response surface for the characteristic ignition-delay time across the full range of possible conditions within a high-speed combustor. The data-driven response surface determines the evolution rate of the transported reaction-evolution variable prior to ignition. Post ignition, the detailed thermochemical state and combustion-evolution rate are tabulated as a function of a reduced number of state variables, including 
the reaction-evolution variable, that are transported in the LES. Further detail is in Section II of this paper.

Such an approach can greatly reduce the computational cost, rendering the simulations tractable, and improve the accuracy of large-eddy simulations of autoignition-dominated, high-speed hydrocarbon combustion. For cases in which a detailed chemical kinetics model is valid, it can be used to construct the response surface for the characteristic ignition-dely time, rather than relying on experimental data.

The evolution-variable manifold (EVM) approach has similarities to the flamelet-progress variable approach of Pierce and Moin ${ }^{2}$ and others, but is developed with a different set of assumptions and modeling limitations, primarily targeting the distributed reaction zone (DRZ) regime. Here, the representation of induction, ignition, and heat release processes is valid across a range of relevant thermodynamic states and stoichiometry that is prescribed by the range of conditions used to obtain the experimental data, and the range of validity of the detailed chemical kinetic models.

In previous work, we proposed a numerical flux formulation that is consistent with the thermodynamic state of the evolving gas mixture. ${ }^{3}$ The University of Minnesota US3D code ${ }^{4}$ was modified to implement the EVM approach using this numerical flux function. The Burke et al. ${ }^{5}$ detailed hydrogen-air kinetics model was used to tabulate the thermochemical state of the gas as a function of the density, energy, mixture fraction, and evolution variable. The CFD code was modified to interpolate on this table and then construct all required thermodynamic variables from the tabulated gas state. The table also includes entries for the rate of change of the evolution variable.

Initial results for RANS and large-eddy simulations of the Gamba et al. ${ }^{6}$ reacting hydrogen jet in crossflow experiments showed good agreement with simulations based on the full chemical kinetics model, the experimental data, and previous simulations. The previous simulations had some sensitivity to the numerical time step; subsequent work on the numerical method and evolution variable tabulation significantly improved these results. In the current implementation, it is possible to run the EVM code at the same large implicit time step as the full chemical kinetics model (corresponding to a non-dimensional time step, or CFL number of order $10^{3}$ when running in Reynolds-averaged Navier-Stokes mode).

In the present paper, we have extended the EVM approach to high-speed turbulent combustion of ethylene. In previous work, ${ }^{1}$ Cymbalist and Dimotakis developed a complete description of ethylene ignition and oxidation based on the available experimental data and a chemical-kinetics mechanism for ethylene-air combustion. This model has been tabulated in a form suitable for use in the US3D-EVM CFD code. The goal of the paper is to present results using this framework for ethylene combustion and compare to previous simulations and experimental data.

Available ethylene experiments in high-speed flows are limited. The most widely used experimental configuration is based on the University of Virginia Supersonic Combustion Experiment. ${ }^{7}$ This flow field has been simulated by Potturi and Edwards ${ }^{8}$ using a 22 -species reduced kinetics model for ethylene, and by Chan and Ihme ${ }^{9}$ using a compressible variant of flamelet-progress variable approach. In this paper, we report on the use of US3D-EVM to study this flow and compare the EVM approach to the previous work. We also perform simulations of the Ben-Yakar, Mungal, and Hanson ethylene-injection experiment ${ }^{10}$ to evaluate the EVM approach in a different flow regime.

\section{Brief Description of the EVM Approach}

At the smallest scales, fluid elements undergoing turbulent combustion in the distributed-reaction zone (DRZ) regime that is anticipated in high-speed combustion are modeled as Lagrangian convected unsteady well-stirred reactors (WSR), entraining surrounding fluid with composition vector components $Y_{s, \text { en }}$ for each of the $s$ species, at a rate $\chi$, or

$$
\begin{gathered}
\frac{d Y_{s}}{d t}=\chi\left(Y_{s, \mathrm{en}}-Y_{s}\right)+\dot{\omega}_{Y_{s}}\left(Y_{s}, h, p\right) \\
\frac{d h}{d t}=\frac{1}{\rho} \frac{d p}{d t}+\chi\left(h_{\mathrm{en}}-h\right),
\end{gathered}
$$

where $Y_{s}$ is the $s$-species mass fraction vector, $h$ and $p$ are the static enthalpy and pressure, $t$ is the reactor time, and $\dot{\omega}_{Y_{s}}$ the $s$-species mass-fraction production rate.

These equations are used to tabulate the post-ignition detailed composition and overall reaction-evolution rate of a fluid element in the DRZ regime, as a function of four variables that include the thermodynamic state in terms of two variables, $[h, p]$ or $[e, \rho]$, a reaction-evolution variable $\tau$, and a conserved mixture fraction 
$\mathcal{Z}$. The species mass-fraction vector components $Y_{s}$ and the Lagrangian fluid element reaction-evolution rate is retrieved during run time as a function of $[e, \rho, \mathcal{Z}, \tau]$.

Briefly describing the EVM framework, the overall reaction rate $\zeta$ of a convected fluid element is partitioned into an induction-evolution rate $\zeta_{\mathrm{i}}$ and a combustion-evolution rate $\zeta_{\mathrm{c}}$. For ethylene combustion, the combustion-evolution rate is determined from the rate of product formation using detailed chemical kinetics, while the induction-evolution rate is determined using shock-tube ignition-delay data in an expression of the form,

$$
\zeta_{\mathrm{i}}=\log \left(\frac{\kappa}{\tau_{0}}\right) \frac{\tau}{t_{\mathrm{d}, \mathrm{c}}},
$$

where $\kappa$ is the value of $\tau$ at ignition, $\tau_{0}$ is the background value of the reaction-evolution variable. $t_{\mathrm{d}, \mathrm{c}}$ is the characteristic ignition-delay time that can be expressed as a function of temperature, pressure, and fuel and oxidizer concentrations,

$$
\frac{1}{t_{\mathrm{d}, \mathrm{c}}}=\zeta_{0} \theta^{a} \pi^{b} \exp \left(\frac{-\alpha(1+\eta \pi)}{\theta}\right) Y_{\mathrm{C}_{2} \mathrm{H}_{4}}^{c} Y_{\mathrm{O} 2}^{d},
$$

where $\theta=T / 1000 \mathrm{~K}, \pi=p / 1 \mathrm{~atm}$. The fit parameters, $\left[\zeta_{0}, a, b, \alpha, \eta, c, d\right]$, are determined from experimental data using an iterative fitting process. For ethylene, the response surface for $1 / t_{\mathrm{d}, \mathrm{c}}$ was determined using 900 experimental data points, is shown in Fig. 1 (see Ref. 1). The resulting fit parameters are shown in Table 2.

Table 2. Fit coefficients

\begin{tabular}{cc}
\hline$\zeta_{0}$ & $7.81 \times 10^{15} \mathrm{~s}^{-1}$ \\
\hline$a$ & -10.6 \\
\hline$b$ & 0.628 \\
$\beta$ & 30.0 \\
\hline$\eta$ & -0.0026 \\
\hline$c$ & 0.0564 \\
\hline$d$ & 0.797 \\
\hline
\end{tabular}

The solid surface with the overlaid mesh represents the region of validity in $[\theta, \pi]$-space of the expression for $1 / t_{\mathrm{d}, \mathrm{c}}$, while the points are the experimental data on which the fitted surface is based.

Post-ignition, the tables are populated by the Lagrangian, unsteady WSR equations, whose solution can be shown to be approximately path independent. ${ }^{1}$ The University of California San Diego detailed chemicalkinetic mechanism ${ }^{11}$ (50 species, 250 reactions) was used to determine the chemical source terms, $\dot{\omega}_{Y_{s}}$. The coordinates of the tables were selected as $[e, \rho, \mathcal{Z}, \tau]$ to facilitate lookup and integration with the compressible large-eddy simulation code.

For illustrative purposes, Fig. 2 shows slices of the carbon monoxide mass fraction, $Y_{\mathrm{CO}}$, extracted from the four-dimensional $[e, \rho, \mathcal{Z}, \tau]$ manifold. The three-dimensional subspace was generated by setting the value of the fourth coordinate to a fixed value. On the left, the fourth coordinate is defined as the $e$ axis, which is fixed to a constant value of $1.6 \times 10^{6} \mathrm{~J} / \mathrm{kg}$. On the right, the fourth coordinate is defined as the $\rho$ axis, which is fixed to a constant value of $0.5 \mathrm{~kg} / \mathrm{m}^{3}$.

\section{EVM-LES Implementation}

There are many possible ways to connect the EVM table to a large-eddy simulation. In this section, we describe how the thermodynamic state and transport properties of the gas mixture are computed, which variables are tabulated, and how the diffusive enthalpy transport term is evaluated.

First, consider the calculation of the mixture state. Rather than tabulating all possible thermodynamic variables and transport properties (e.g., Saghafian et al. ${ }^{12}$ in the context of a compressible flamelet-progress variable model), we follow the approach suggested by Oevermann. ${ }^{13}$ That is, we tabulate the mass fractions obtained from the detailed chemistry model and compute the local thermodynamic state from the deduced mass fractions, density, and energy. This requires the use of thermodynamic data for the chemical species, such as the Gordon-McBride enthalpy curve fits. ${ }^{14}$ Then, we can compute the mixture transport properties from the thermodynamic state and tabulated mass fractions. 


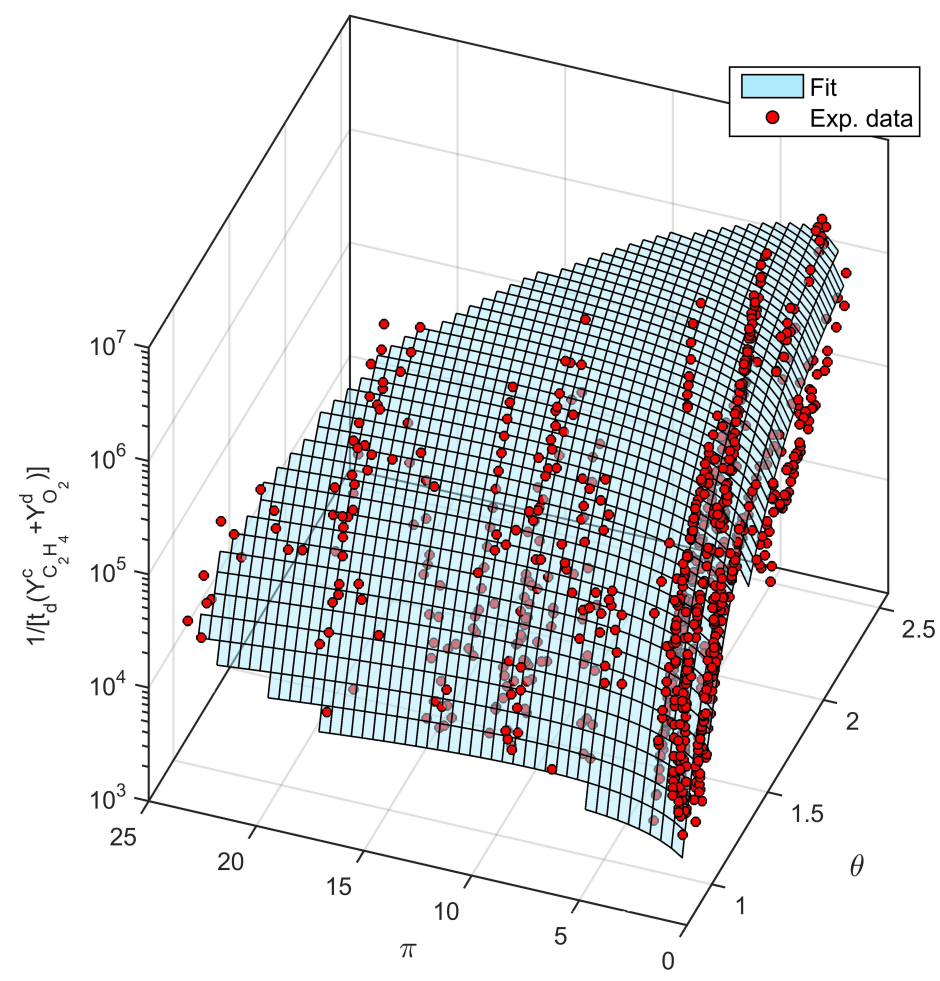

Figure 1. Data-driven reciprocal ignition-delay surface.
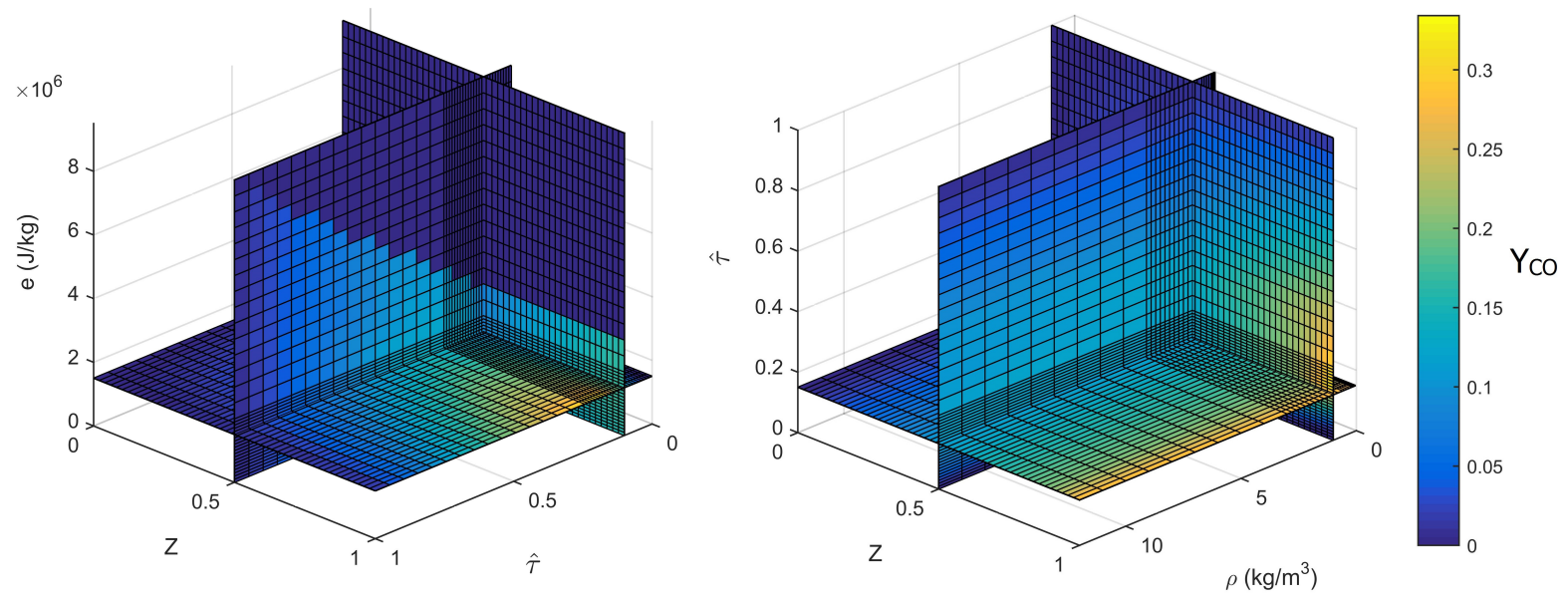

Figure 2. Slices of the carbon monoxide mass fraction, $Y_{\mathrm{CO}}$, extracted from the four-dimensional $[e, \rho, \mathcal{Z}, \tau]$ manifold. 
For hydrocarbon combustion models involving many chemical species, only species that are present at significant levels need to be tabulated. For example, radicals that are present at very low levels and are critical to the induction process, need not be tabulated because they do not appreciably affect the thermodynamic state. For example, for the 50-species San Diego ethylene model used in the present work, 25 species are tabulated.

One additional term requires attention. In the energy conservation equation, the diffusive enthalpy flux

$$
\rho D \sum_{s} h_{s} \frac{\partial Y_{s}}{\partial x_{j}}
$$

involves the computation of the species enthalpy and gradients of the detailed model mass fractions. Quinlan et al. ${ }^{15}$ propose a simplification to this term of the form:

$$
\rho D \sum_{s} h_{s} \frac{\partial Y_{s}}{\partial \mathcal{Z}} \frac{\partial \mathcal{Z}}{\partial x_{j}}
$$

Then just the single term $\sum_{s} h_{s} \frac{\partial Y_{s}}{\partial \mathcal{Z}}$ can be tabulated. Their a priori analysis shows relatively minor differences using this approach compared to the full expression. In our simulations, we have found that this approximation is not sufficiently accurate, resulting in spurious temperature variations and poor numerical stability. Thus, in the work presented here, we use the full expression, (5). This requires storage of the interpolated values of $Y_{s}$ at element centroids and the computation of the $Y_{s}$ gradients.

Also note that we solve for $\mathcal{C}$, the product mass fraction, but we tabulate the product state with $\tau=\mathcal{C} / \mathcal{C}_{e q}$ However, $\mathcal{C}_{e q}$ is a function of $\rho, e, \mathcal{Z}$ only, and can be obtained from the table by extracting the product mass fractions at $[\rho, e, \mathcal{Z}, \tau=1]$. Then, the local value of $\tau$ can be computed, which then is used to obtain the local mixture state.

The EVM table is four-dimensional, and a general search in this domain would be expensive. However, it is stored as an ordered array, and the relationship between the values of the variables $(\rho, e, \mathcal{Z}, \tau)$ and their indices are known. Thus, simple integer math produces the bounding indices of the location in the table, and then a tetra-linear interpolation is performed to obtain the values of $\zeta$, its derivatives with respect the tabulation variables (computed using finite-differences on the EVM table), and the mass fractions. The table is stored as single precision (32-bit) real values to reduce its size.

Thus, to summarize, the LES provides the value of $[e, \rho, \mathcal{Z}, \tau]$ at each time step to the EVM table, that then returns the detailed fluid composition $Y_{s}$ and overall reaction evolution rate $\zeta$, tabulated a priori using the Lagrangian well-stirred reactor model and the experimental data-driven induction-evolution model. Figure 3 illustrates the exchange of information between the LES and EVM modules during runtime.

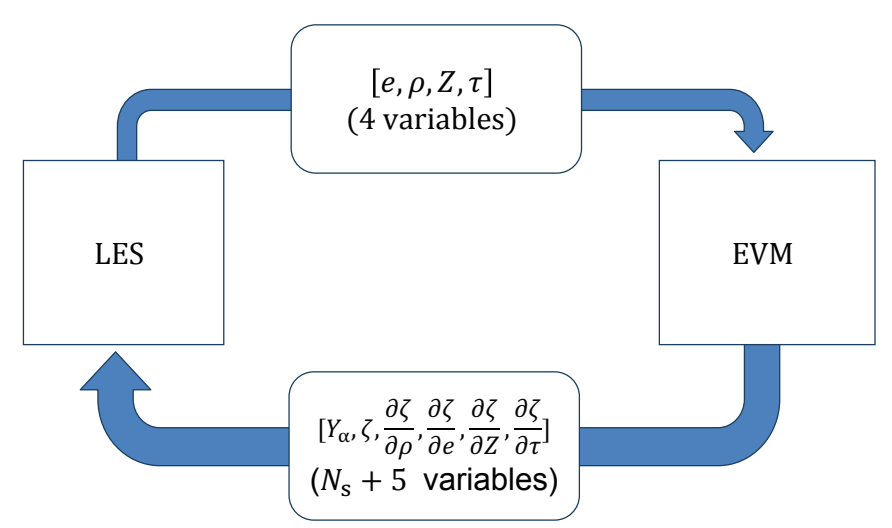

Figure 3. Exchange of information between the LES and EVM modules during runtime 


\section{Governing Equations}

In this section, we discuss the governing equations used for the evolution- variable manifold approach. As discussed above, the EVM table uniquely determines the thermo-chemical state from the density, energy, mixture fraction, $\mathcal{Z}$, and induction-evolution variable, $\tau$. As stated above, $\tau$ is defined as the ratio of the reaction product mass fraction to its equilibrium mass fraction

$$
\tau=\frac{\mathcal{C}}{\mathcal{C}_{\mathrm{eq}}}
$$

where $\mathcal{C}$ represents the product mass fraction. For ethylene, we choose $\mathcal{C}$ to be the sum of the product mass fractions

$$
\mathcal{C}=Y_{\mathrm{CO}}+Y_{\mathrm{CO}_{2}}+Y_{\mathrm{H}_{2} \mathrm{O}}+Y_{\mathrm{H}_{2}}
$$

Note that $\tau$ is not a conserved variable, and we must solve for the progress variable density. Thus, the conservation equations for the density, mixture fraction density, and product fraction density are:

$$
\begin{aligned}
\frac{\partial \rho}{\partial t}+\frac{\partial}{\partial x_{j}}\left(\rho u_{j}\right) & =0 \\
\frac{\partial \rho \mathcal{Z}}{\partial t}+\frac{\partial}{\partial x_{j}}\left(\rho \mathcal{Z}\left(u_{j}+v_{\mathcal{Z}}\right)\right) & =0 \\
\frac{\partial \rho \mathcal{C}}{\partial t}+\frac{\partial}{\partial x_{j}}\left(\rho \mathcal{C}\left(u_{j}+v_{\mathcal{C} j}\right)\right) & =\rho \dot{\omega}_{\mathcal{C}}
\end{aligned}
$$

These are solved along with the momentum and total energy conservation equations. Here, $v_{\mathcal{Z} j}$ and $v_{\mathcal{C} j}$ are the mass diffusion velocities due to molecular and turbulent transport.

The source term on the $\rho \mathcal{C}$ equation represents the rate of production of the product, $\dot{\omega}_{\mathcal{C}}=\mathcal{C}_{\text {eq }} \zeta$. where $\zeta$ is the rate of production of the induction-evolution variable; $\zeta$ is obtained from Eq. (3) prior to ignition, and from the product source term from the detailed mechanism after combustion has been initiated.

Here, $\rho \mathcal{Z}$ is the density of the elements comprising the fuel and its reaction products; we can define a second partial density of the gas mixture as $\rho \mathcal{X}=\rho-\rho \mathcal{Z}$, which is the density of the gas mixture that is that is not composed of fuel and its reaction products. The conservation equation for $\rho \mathcal{X}$ is

$$
\frac{\partial \rho \mathcal{X}}{\partial t}+\frac{\partial}{\partial x_{j}}\left(\rho \mathcal{X}\left(u_{j}+v_{\mathcal{X}}\right)\right)=0
$$

with $v_{\mathcal{X}}=-v_{\mathcal{Z}}$ for mass conservation. In the following, we use the $\rho \mathcal{X}$ variable because it simplifies the derivation of the flux Jacobian and the formulation of the numerical flux function. Also, the resulting flux Jacobian has a form similar to that obtained for a mixture of reacting gases. ${ }^{16}$

Thus, the vector of conserved quantities and the corresponding convective flux vector for a threedimensional flow are

$$
\begin{aligned}
U & =(\rho \mathcal{X}, \rho \mathcal{Z}, \rho \mathcal{C}, \rho u, \rho v, \rho w, E)^{T} \\
F & =\left(\rho \mathcal{X} u^{\prime}, \rho \mathcal{Z} u^{\prime}, \rho \mathcal{C} u^{\prime}, \rho u u^{\prime}+p n_{x}, \rho v u^{\prime}+p n_{y}, \rho w u^{\prime}+p n_{z},(E+p) u^{\prime}\right)^{T}
\end{aligned}
$$

where $u^{\prime}=\vec{u} \cdot \hat{n}$ is the velocity normal to a cell face with unit normal vector $\hat{n}$. Here, $E$ is the total energy per unit volume, $E=\rho\left(e+\frac{1}{2} \vec{u} \cdot \vec{u}\right)$. The detailed thermo-chemical state is obtained from the evolution-variable manifold (table) as discussed above and in detail by Cymbalist and Dimotakis. ${ }^{1}$

\section{Numerical Method}

In this section, we discuss an approach to obtaining a self-consistent numerical flux function for the evolution-variable manifold approach based on a set of tabulated thermodynamic data. First, we develop the convective flux Jacobian required for an upwind-biased flux, then discuss an approach for obtaining second-order accurate fluxes, and finally provide a brief description of a low-dissipation centered flux function suitable for highly compressible flows. 


\section{V.A. Upwind Numerical Flux Formulation}

Let us first consider an upwind flux formulation such as modified Steger-Warming flux-vector splitting ${ }^{17}$ or Roe flux-difference splitting. ${ }^{18}$ Such a numerical flux is appropriate for Reynolds-averaged Navier-Stokes (RANS) simulations of high-speed combustion flows. For LES applications, we use the dissipative portion of the upwind flux to stabilize a low-dissipation centered flux.

For the upwind flux, we must diagonalize the flux Jacobian, $\frac{\partial F}{\partial U}$. This is straight-forward except for the derivatives of the pressure with respect to the conserved variables. Following the approach in Ref. 16, it can be shown that

$$
\begin{aligned}
\frac{\partial F}{\partial U}=R^{-1} \Lambda R= & \left(\begin{array}{c}
\mathcal{X} \hat{\lambda} / a^{2} \\
\mathcal{Z} \hat{\lambda} / a^{2} \\
\mathcal{C} \hat{\lambda} / a^{2} \\
\left(u \hat{\lambda}+a n_{x} \tilde{\lambda}\right) / a^{2} \\
\left(v \hat{\lambda}+a n_{y} \tilde{\lambda}\right) / a^{2} \\
\left(w \hat{\lambda}+a n_{z} \tilde{\lambda}\right) / a^{2} \\
\left(h_{o} \hat{\lambda}+a u^{\prime} \tilde{\lambda}\right) / a^{2}
\end{array}\right) \\
+ & \left(\begin{array}{c}
p_{\rho \mathcal{X}} \\
\mathcal{X} \tilde{\lambda} / a \\
\mathcal{Z} \tilde{\lambda} / a \\
\mathcal{C} \tilde{\lambda} / a \\
u \tilde{\lambda} / a+n_{x} \hat{\lambda} \\
v \tilde{\lambda} / a+n_{y} \hat{\lambda} \\
w \tilde{\lambda} / a+n_{z} \hat{\lambda} \\
h_{o} \tilde{\lambda} / a+u^{\prime} \hat{\lambda}
\end{array}\right)
\end{aligned}
$$

Where the diagonal matrix of eigenvalues is given as

$$
\Lambda=\operatorname{diag}\left(\lambda, \lambda, \lambda, \lambda^{+}, \lambda, \lambda, \lambda^{-}\right)
$$

where $\lambda=u^{\prime}, \lambda^{+}=u^{\prime}+a, \lambda^{-}=u^{\prime}-a, a$ is the speed of sound, and $\tilde{\lambda}$ and $\hat{\lambda}$ are defined as

$$
\tilde{\lambda}=\frac{1}{2}\left(\lambda^{+}-\lambda^{-}\right), \quad \hat{\lambda}=\frac{1}{2}\left(\lambda^{+}+\lambda^{-}-2 \lambda\right)
$$

and $h_{o}$ is the total enthalpy of the gas mixture, $h_{o}=e+p / \rho+\frac{1}{2} \vec{u} \cdot \vec{u}$.

The pressure derivatives

$$
p_{\rho \mathcal{X}}=\frac{\partial p}{\partial \rho \mathcal{X}}, \quad p_{\rho \mathcal{Z}}=\frac{\partial p}{\partial \rho \mathcal{Z}}, \quad p_{E}=\frac{\partial p}{\partial E}
$$

must be computed holding the conserved variables, $U$, fixed. For a multi-species mixture of thermally-perfect gases, the pressure may be written as:

$$
p=\rho \sum_{s} Y_{s} \frac{\hat{R}}{M_{s}} T
$$

where the summation is over all species, and $Y_{s}$ is the mass fraction of species $s$. (Here, $Y_{s}$ denotes the species stored in the EVM table.) With the EVM approach, we tabulate the mass fractions and construct the pressure from the thermodynamic state $(\rho$ and $e$ ). However, it is not possible to explicitly determine how the species state varies with the conserved variables, and thus exact expressions for the pressure derivatives cannot be obtained. Instead, we assume that the mass fractions are frozen when taking the pressure derivatives, and then closed-form expressions can be obtained. Thus, the pressure derivatives are:

$$
\left.\frac{\partial p}{\partial \rho \mathcal{X}}\right|_{\rho \mathcal{Z}, \rho \mathcal{C}, \rho \vec{u}, E}=\left.\frac{\partial p}{\partial \rho \mathcal{Z}}\right|_{\rho \mathcal{X}, \rho \mathcal{C}, \rho \vec{u}, E}=\bar{R} T+\frac{\bar{R}}{c_{v}}\left(-e+\frac{1}{2} \vec{u} \cdot \vec{u}\right)
$$

where $e, R$, and $c_{v}$ are the mixture internal energy per unit mass, gas constant, and specific heat:

$$
e=\sum_{s} Y_{s} e_{s}, \quad \bar{R}=\sum_{s} Y_{s} \frac{\hat{R}}{M_{s}} T, \quad c_{v}=\sum_{s} Y_{s} c_{v s}
$$


Thus, we have a closed form for the convective flux Jacobian and we can formulate an upwind flux function. For example, the Roe flux is ${ }^{18}$

$$
F_{i+1 / 2}=\frac{1}{2}\left(F_{i}+F_{i+1}\right)-\frac{1}{2} \tilde{R}^{-1}|\tilde{\Lambda}| \tilde{R}\left(U_{i+1}-U_{i}\right)
$$

where the tilde-variables are Roe-averaged using the left and right data. For modified Steger-Warming flux vector splitting, ${ }^{17}$ the convective fluxes may be written as

$$
F_{i+1 / 2}=A_{i+1 / 2}^{+} U^{L}+A_{i+1 / 2}^{-} U^{R}
$$

Where $A^{ \pm}$are the Jacobians evaluated using the face data and the positive and negative eigenvalues, respectively.

The quantities $U^{L, R}$ are the conserved variables evaluated at the face using left- and right-biased data. For highly compressible flows, it is better to evaluate the primitive variables at the face and reconstruct the face values of the conserved variables. Thus, with MUSCL-type limiting, ${ }^{19}$ we have

$$
\begin{aligned}
& V_{i+1 / 2}^{L}=V_{i}+\frac{1}{2} \lim \left(V_{i+1}-V_{i}, V_{i}-V_{i-1}\right) \\
& V_{i+1 / 2}^{R}=V_{i+1}-\frac{1}{2} \lim \left(V_{i+2}-V_{i+1}, V_{i+1}-V_{i}\right)
\end{aligned}
$$

where $V=(\rho \mathcal{X}, \rho \mathcal{Z}, \rho \mathcal{C}, u, v, w, p)^{T}$. It is trivial to obtain all of the conserved variables from the primitives, except for $E^{L, R}$.

We find that the solution is sensitive to how the energy at the face is reconstructed. The most accurate approach is to store the mass fractions, $Y_{s}$, obtained from the table for every grid element. Then, these mass fractions are limited as in Eqs. (24) and (25) to form the mixture-averaged gas constant at the face, $\bar{R}^{L, R}$. Then the face temperature is obtained from the face values of density and pressure.

$$
T^{L}=\frac{p^{L}}{\rho^{L} \bar{R}^{L}}, \quad T^{R}=\frac{p^{R}}{\rho^{R} \bar{R}^{R}}
$$

Now the face state is uniquely specified and the energy can be computed from $Y_{s}^{L, R}$ and $T^{L, R}$.

Other less accurate energy reconstruction approaches were tested with poor results. These methods included assuming that the gas state is frozen between the upwind element centroid and the face, and reconstructing the internal energy using a Taylor series for the energy and its derivatives. These approaches resulted in spurious non-monotone temperature variations across reaction fronts and the implicit time integration was less stable. We concluded that the complete energy reconstruction is required.

\section{V.B. Kinetic Energy Consistent Flux}

The LES results presented below were computed with a fourth-order accurate centered flux function that has been shown to have low levels of dissipation. The flux is formulated so that it is discretely consistent with the compressible kinetic energy equation. ${ }^{20}$ The dissipative component of the modified Steger-Warming flux discussed above is added to provide dissipation near shock waves and other strong gradients. The Ducros sensor ${ }^{21}$ is used to activate the dissipative flux through a weight function, $\alpha_{d}$. The numerical flux may be written as

$$
F_{i+1 / 2}=F_{\mathrm{KEC}, i+1 / 2}-\alpha_{d} \frac{1}{2} R^{-1}|\Lambda| R\left(U_{i+1}-U_{i}\right),
$$

where the centered flux is

$$
F_{\mathrm{KEC}, i+1 / 2}=\left(\begin{array}{c}
\bar{\rho} \overline{\mathcal{X}} \bar{u}^{\prime} \\
\bar{\rho} \overline{\mathcal{Z}} \bar{u}^{\prime} \\
\bar{\rho} \overline{\mathcal{C}} \bar{u}^{\prime} \\
\bar{\rho} \bar{u}^{\prime} \bar{u}+\bar{p} n_{x} \\
\bar{\rho} \bar{u}^{\prime} \bar{v}+\bar{p} n_{y} \\
\bar{\rho} \bar{u}^{\prime} \bar{w}+\bar{p} n_{z} \\
\bar{\rho} \bar{u}^{\prime}(\bar{e}+\bar{k})+\bar{p} \bar{u}^{\prime}
\end{array}\right)
$$


Here the bars indicate and average between the left and right data, such as $\bar{\rho}=\frac{1}{2}\left(\rho^{L}+\rho^{R}\right)$; for the kinetic energy we use

$$
\bar{k}=\frac{1}{2}\left(u^{L} u^{R}+v^{L} v^{R}+w^{L} w^{R}\right)
$$

For second order, the nearest data are used, for example $\bar{\rho}=\frac{1}{2}\left(\rho_{i}+\rho_{i+1}\right)$; for a fourth or higher-order flux formulation, gradients may be used to obtain a more accurate representation of the face variables. For the fourth-order flux used in this work, the left and right states are obtained using:

$$
\begin{gathered}
\phi_{i+1 / 2}^{L}=\phi_{i}+\beta \nabla \phi_{i} \cdot\left(\vec{x}_{i+1 / 2}^{f}-\vec{x}_{i}\right) \\
\phi_{i+1 / 2}^{R}=\phi_{i+1}+\beta \nabla \phi_{i+1} \cdot\left(\vec{x}_{i+1 / 2}^{f}-\vec{x}_{i+1}\right)
\end{gathered}
$$

with $\beta=2 / 3$. Again, as with the upwind-biased flux, we construct $\bar{e}$ from the face values of pressure, gas constant, and the mass fractions.

\section{V.C. Time Integration}

The results presented below are based on a grid that resolves the near-wall boundary layer, rather than using a wall model and coarse near-wall grid spacing. This approach imposes stringent time-step limitations; also, the source term on the reaction product equation may be large, imposing an additional restriction on explicit time integration methods. Therefore, implicit time integration methods are used. For RANS simulations, the data-parallel line-relaxation method $^{22}$ is used, and for wall-modeled large-eddy simulations (WM-LES), a second-order accurate version of an implicit point-relaxation method ${ }^{23}$ is employed. As stated above, the derivatives of the induction-evolution variable source term, $\zeta$, are tabulated and are used to form the Jacobian of the source term for use in the implicit operator.

\section{Simulations of the Ben-Yakar et al. ethylene combustion experiments}

The EVM approach was used to simulate the ethylene normal injection experiments of Ben-Yakar, Mungal, and Hanson. ${ }^{10}$ The computational domain was chosen to resolve the incoming boundary layer; the injection plenum was gridded, and embedded refinement regions were included near the jet, in the jet interaction region, and in the jet plume. The near-wall grid was clustered to better resolve the boundary layer, with the near-wall spacing corresponding to $y^{+} \simeq 1$.

Figure 4 shows the computational domain, along with a temperature isosurface to visualize the jet plume. Figure 5 shows two images of the grid, highlighting the embedded refinement regions in the outflow plane and around the injector. Note the use of 3-point and 5-point grid singularities that allow the grid resolution to smoothly diminish away from the jet and plume. The grid was generated using the GoHypersonic Inc. LINK3D software. ${ }^{24}$ There are a total of 14.1 million hexahedral elements in the grid. This grid was refined by approximately a factor of two in each direction, resulting in a finer grid with 107.4 million hexahedral elements.

Flow conditions are taken from Ben-Yakar et al. ${ }^{10}$ as $T_{\infty}=1290 \mathrm{~K}, p_{\infty}=32.4 \mathrm{kPa}$, and $u_{\infty}=2360 \mathrm{~m} / \mathrm{s}$. This corresponds to a free-stream density of $\rho_{\infty}=0.0875 \mathrm{~kg} / \mathrm{m}^{3}$, Mach number of 3.38 and a Reynolds number of 8100 based on the $d_{\mathrm{j}}=2 \mathrm{~mm}$ diameter jet and freestream conditions. The ethylene plenum stagnation pressure and temperature were set to $1.00 \mathrm{MPa}$ and $298 \mathrm{~K}$. An isothermal surface temperature boundary condition at $300 \mathrm{~K}$ was assumed to represent the short-duration operation of the shock tube experiment.

The simulations were first run in RANS mode (using the Spalart-Allmaras one-equation turbulence model $^{25}$ and the Catris-Aupoix compressibility correction ${ }^{26}$ ) with a second-order accurate upwind flux method and implicit time integration with large time steps. Then the simulations were restarted as a wall-modeled LES using the Improved Delayed Detached Eddy Simulation (IDDES) method of Shur et al. ${ }^{27}$ and the fourth-order accurate kinetic energy consistent low-dissipation fluxes and second-order accurate implicit time integration. IDDES in combination with low-dissipation fluxes has been shown to compare well with jet in supersonic crossflow mixing experiments. ${ }^{28}$ Effectively, it uses the Spalart-Allmaras RANS model as a wall model, and a Smagorinsky-like subgrid-scale model outside the wall boundary layer. For the LES, much smaller time steps of about $\Delta t \simeq 2 \mathrm{~ns}$ were used (corresponding to a non-dimensional CFL of about $10)$. 


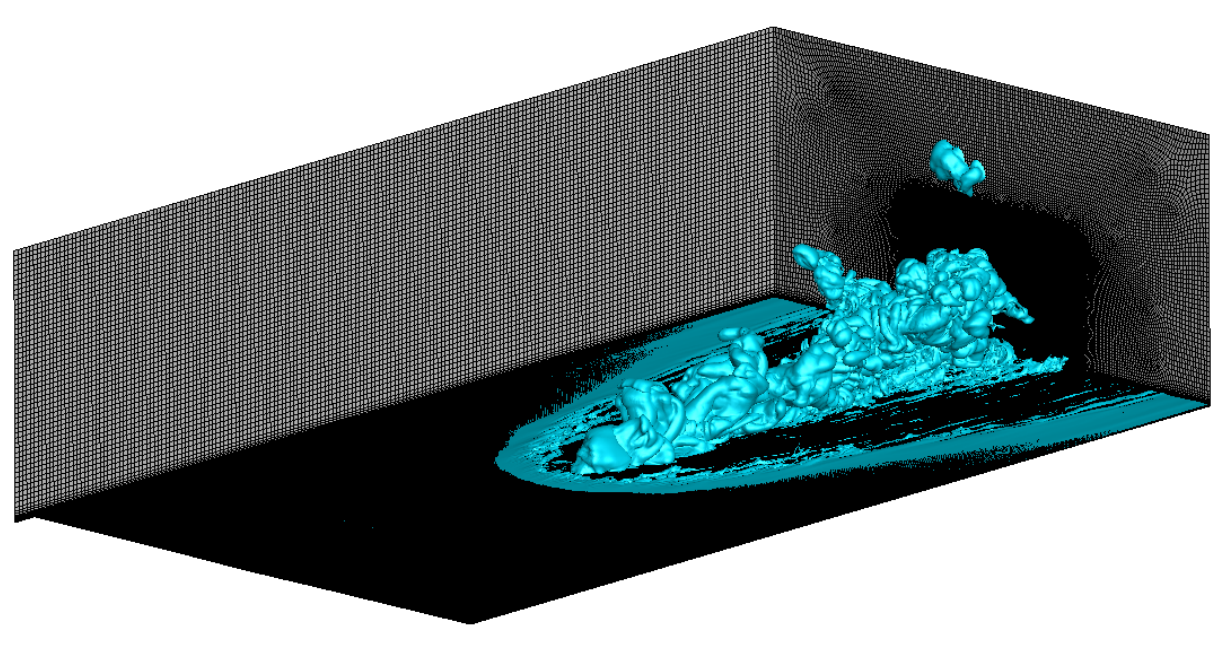

Figure 4. Flow field showing iso-surface of the temperature $(T=1100 \mathrm{~K})$ to visualize the flow field and solution domain.
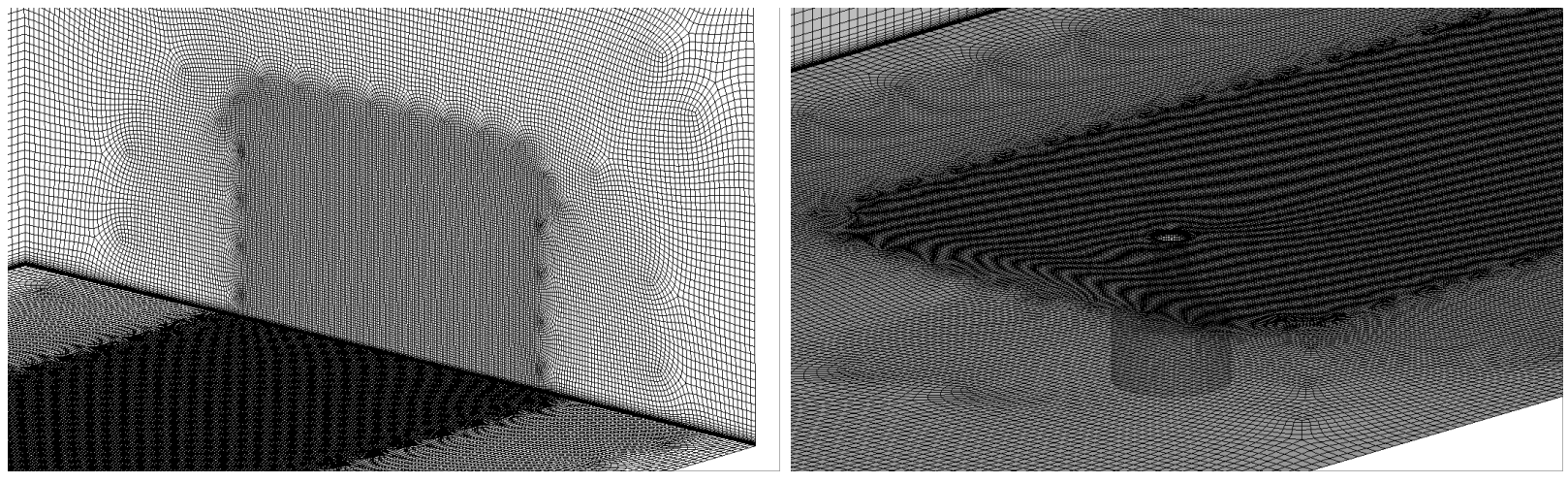

Figure 5. Sections of the 14 million element computational grid used for the Ben-Yakar, Mungal, and Hanson simulations; surface grid near injector (left) and surface and outflow grid (right). 
Figure 6 plots contours of instantaneous temperature, mixture fraction, and product source term for the EVM-LES of the Ben-Yakar et al. experiment on the two grids. Here, the solution on the centerline, nearwall plane, and outflow plae are plotted. There is a very large qualitative difference between the simulations, with the coarse grid producing only a small level of unsteadiness and a coherent plume. The injectant gas is confined to the plume, and reactions only occur at the edges of the jet. The solution on the higher-resolution grid is much more unsteady and intermittent, with a large range of length scales. This results in much more dispersion and mixing of $\mathcal{Z}$. Note that $\zeta$ is active at the jet boundaries, but also in the region underneath the jet where the counter-rotating vortex pair has caused additional mixing of fuel and oxidizer. Note that there is non-zero production well off the centerline due to entrainment and mixing in the horeshoe vortices generated by the interaction with the jet. Thus, the higher grid resolution EVM-LES resolves the small scales of combustion in regimes that include distributed-reaction zones and thin-reaction zones.

Figure 7 plots the simulated $\mathrm{OH}$ planar laser-induced fluorescence (PLIF) signal computed from the results. ${ }^{29}$ Again note the highly coherent signal for the small coarse, and the intermittent signal obtained with the higher-resolution grid. The latter image is similar to that obtained by Ben-Yakar et al.; ${ }^{10}$ though since the experiments only show a single image, this comparison can only be qualitative. The results obtained using the higher-resolution grid show that the LES-EVM approach can capture experimentally observed features of autoignition-dominated combustion in high-speed/supersonic flows. We note, however, that a further increase in resolution by a factor of 2 may have produced different results yet, but was out of computational reach.

Figure 8 plots mass fraction contours of two of the 25 species used in the EVM-LES ethylene simulations, $\mathrm{CH}$ and $\mathrm{CH}_{3}$, on the large grid. Note that the $\mathrm{CH}$ radical has a variation similar to that of $\mathrm{OH}$, while $\mathrm{CH}_{3}$ correlates well with $\zeta$. Because the present EVM-LES approach tabulates all key species, these can be directly extracted from the simulations.

Interestingly, the Ben-Yakar et al. case appears to be particularly sensitive to grid resolution; previous simulations ${ }^{3}$ of the hydrogen injection experiments of Gamba, ${ }^{6}$ show that highly unsteady results are obtained on small grids, similar to the 14.1 million element grid used here.

\section{University of Virginia Ethylene Simulations}

The EVM-LES approach was also used to simulate the University of Virgina ethylene combustion experiments operating in Configuration E. ${ }^{7,8}$ The combustor involves injection of ethylene into an approximately Mach 2 flow with a stagnation temperature of $1200 \mathrm{~K}$. The constant cross-section isolator is $1 \times 1.5 \mathrm{inch}$ in dimension; divergence is added just upstream of the fuel injection location. The combustor is approximately 35 inches in length. Ethylene is injected through 5 small normal injectors upstream of a flame-holding cavity. Extensive measurements of this flow field have been made, though at the present time we have not obtained time-averaged data so as to make direct comparisons with the experiments.

A similar computational setup was used to that of Potturi and Edwards ${ }^{8}$ who simulated this experiment with an 22-species reduced model using similar numerical methods to those used in the present work. Here, a 215 million hexahedral element grid was generated with LINK3D. ${ }^{24}$ The region inside the injectors was gridded, and typical near-wall spacings of $2 \mu \mathrm{m}$ were used to ensure $y^{+}<1$ at all walls (except in the injectors where the boundary layers are extremely thin due to the high ethylene density). The grid is nearly isotropic in the cavity so as to produce reliable LES results. Figure 9 shows two images to illustrate the grid quality.

The UVa facility nozzle was simulated with RANS to provide inflow data for the EVM-LES simulation. The digital filter technique (Klein et al. ${ }^{30}$ Xie and Castro, ${ }^{31}$ Touber and Sandham ${ }^{32}$ ) was used to generate the synthetic turbulence field. These inflow perturbations are generated using data from the RANS simulation, such that they match the prescribed Reynolds stresses. The mean and fluctuating profiles were then interpolated onto the inflow plane of the EVM-LES simulation. To date, simulations without the synthetic turbulence have been completed, and these are shown below; these will be updated when the full simulations are complete.

The flow was initialized by running the US3D-EVM code in RANS mode to establish an initial flow field; then the the IDDES wall-modeled LES approach was activated, along with fourth-order accurate KEC fluxes and second-order accurate time integration. Time steps corresponding to a CFL of 20 were used (a physical time step of $2.4 \mathrm{~ns}$ ), and about 2 combustor flow-through times have been completed to date (again, without the unsteady synthetic turbulent inflow).

Figures 10 and 11 show instantaneous images of the University of Virginia EVM-LES results. Here, the 

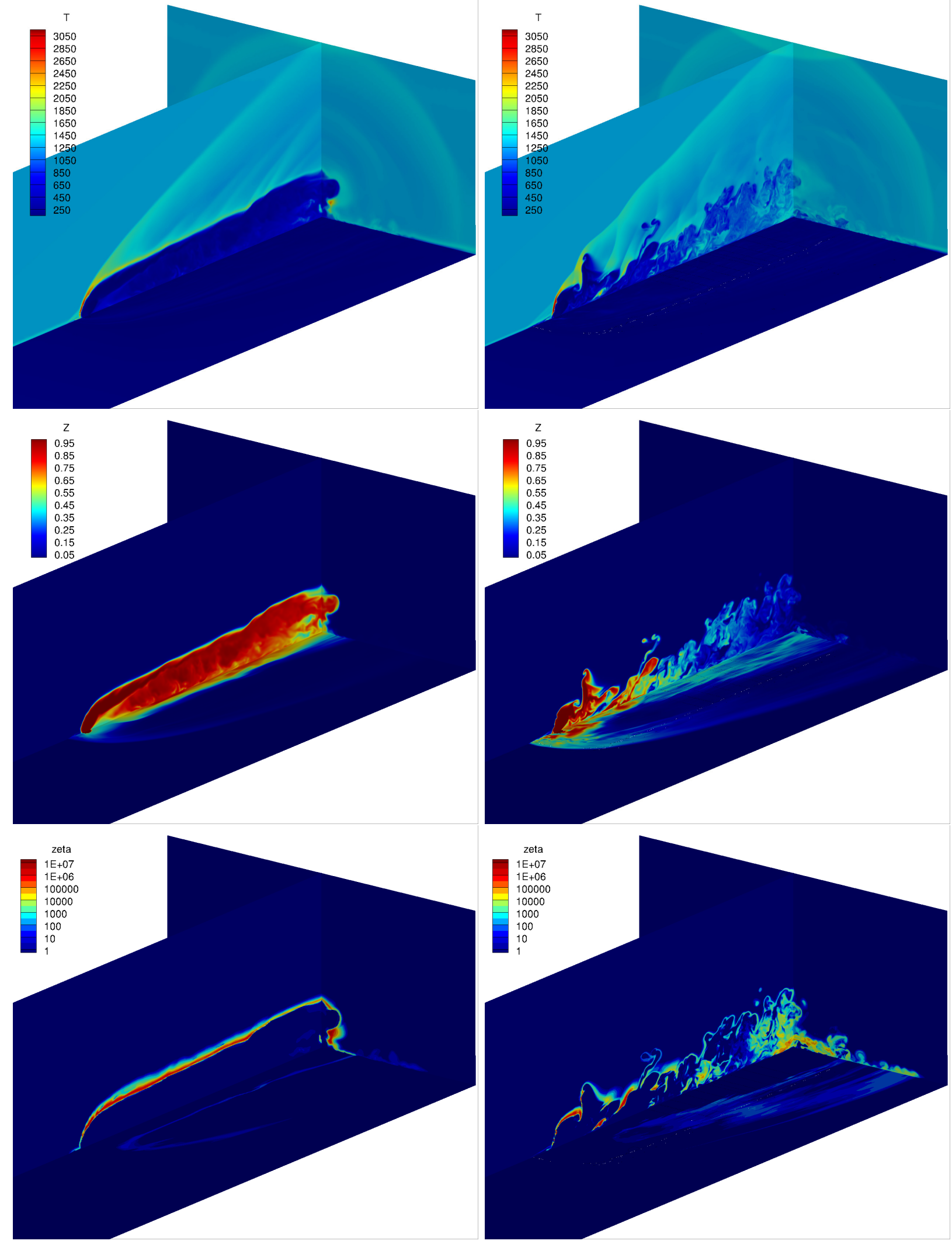

Figure 6. Ben-Yakar simulation results: Temperature (top), mixture fraction $\mathcal{Z}$ (center), and product source term $\zeta$ (bottom); $14.1 \mathrm{M}$ element grid (left) and $107.4 \mathrm{M}$ element grid (right). 

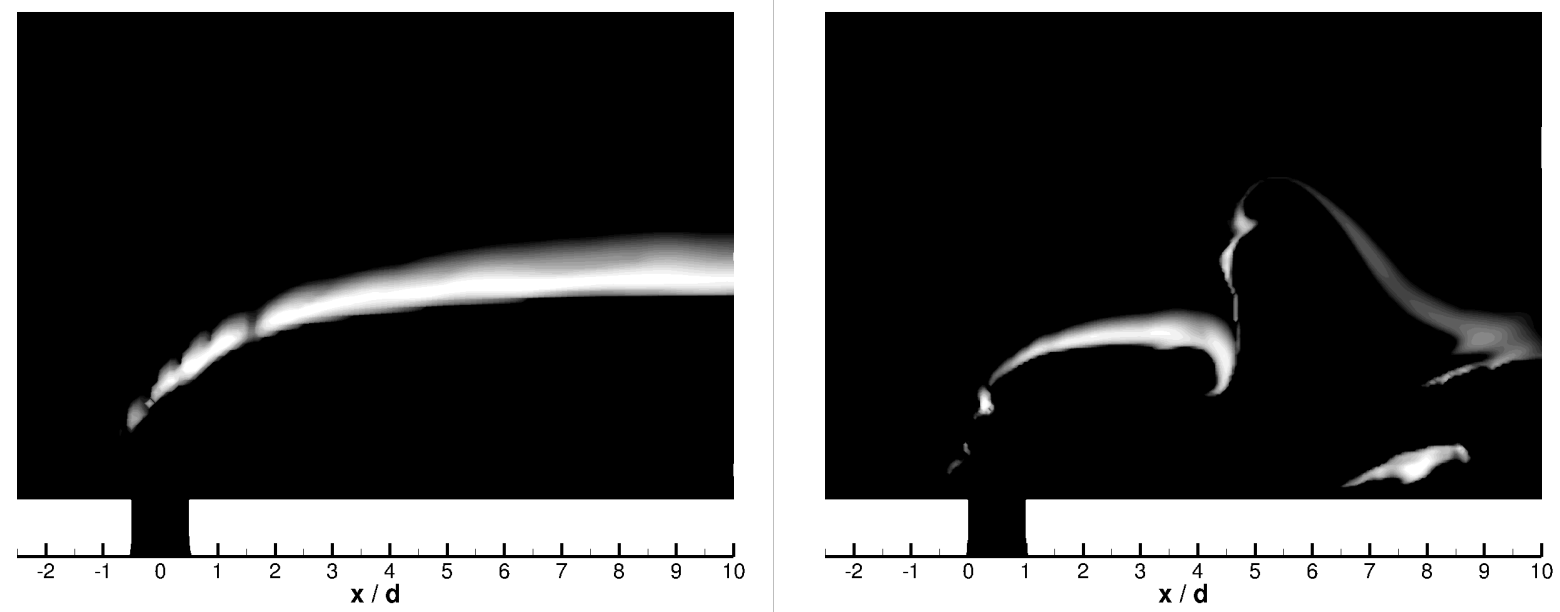

Figure 7. Simulated OH PLIF on the centerline of the Ben-Yakar experiment; $14 \mathrm{M}$ element grid (left) and $107 \mathrm{M}$ element grid (right).

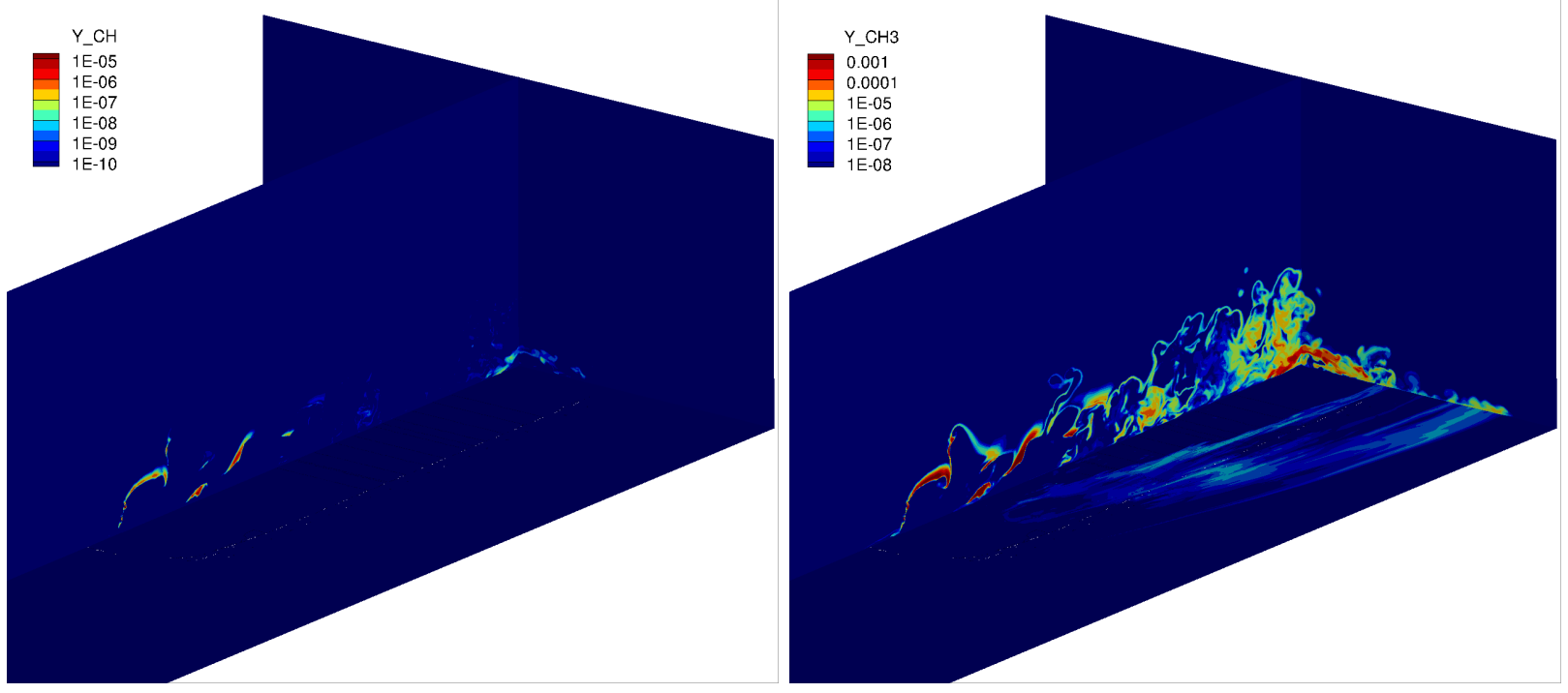

Figure 8. Ben-Yakar simulation results on the $107 \mathrm{M}$ element grid: mass fractions of $\mathrm{CH}$ (left) and $\mathrm{CH}_{3}$ (right). 

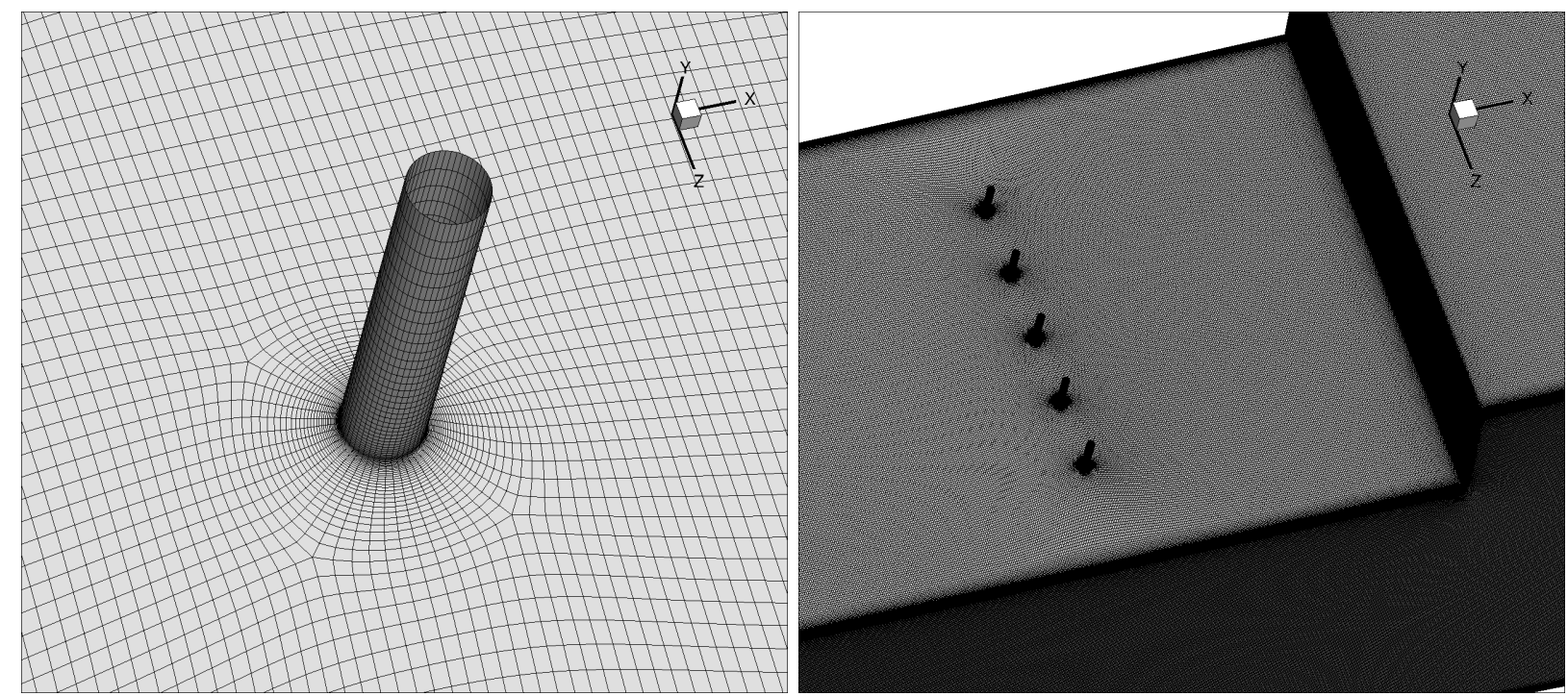

Figure 9. Examples of the 215 million element computational grid used for the University of Virginia combustor simulations.

centerline plane and three cross-section planes are plotted. Qualitatively, the results are similar to those of Potturi and Edwards, with similar levels of temperature and mass fractions in the centerline plane. In this case, $\zeta$ is active in the cavity shear layer, in the aft section of the cavity, and near the upper duct wall where the ethylene continues to mix with the air. The evolution variable, $\tau$, contours show values close to one in the cavity, and then several cavity lengths downstream of the cavity where the combustion is nearing completion. Note that since $\tau=\mathcal{C} / \mathcal{C}_{e q}, \tau=1$ indicates an equilibrium level of product formation. The region of large $\tau$ on the lower combustor wall occurs in regions of very low $\mathcal{Z}$, so are not meaningful.

A notable difference between the present simulations and those of Potturi and Edwards is the variation of pressure. Here, the pressure rise several cavity lengths downstream of the cavity, but in the previous simulations (and the experiments) the pressure continues to rise along the combustor. This may indicate thermal choking, which is not captured in the present simulations; this may be a result of insufficient run time or the use of a supersonic outflow boundary condition. This difference is being investigated.

\section{Conclusions}

The evolution-variable manifold (EVM) approach of Cymbalist and Dimotakis ${ }^{1}$ has been implemented in a computational fluid dynamics code, and wall-modeled large-eddy simulations of reacting ethylene flows have been performed. The LES-EVM approach tabulates the chemical composition and product source term as a function of the density, energy, mixture fraction, and evolution variable. At each time step, the flow state obtained from the LES is used to determine the gas mixture detailed composition, allowing the thermodynamic state and transport properties to be computed. The numerical flux function is designed to be consistent with the thermodynamics, and it is found that particular care must be taken in the construction of the mixture energy for use in computing the total energy flux. In addition, exact treatment of the diffusive enthalpy transport term in the energy equation is found to be important for solution accuracy and stability.

The Ben-Yakar, Mungal, and Hanson ethylene jet in supersonic crossflow experiments were simulated with the LES-EVM approach. Strong sensitivity to the grid resolution was found, with the small grid producing a nearly steady flow, while the large grid giving results qualitatively similar to the experiment and demonstrating large-scale unsteadiness. With the appropriate level of grid resolution, the EVM-LES was able to capture thin reaction zones as well as distributed reaction zones in this flow. The University of Virginia ethylene combustor experiments ${ }^{7}$ were also simulated; initial results are similar to previous computations ${ }^{8}$ obtained with a much more expensive kinetics model. Additional flow simulation time is required to obtain quantitative comparisons with the previous simulations and with experiment. At this stage, the EVM-LES approach shows promise for predicting autoignition-dominated flows relevant to high-speed combustion. 

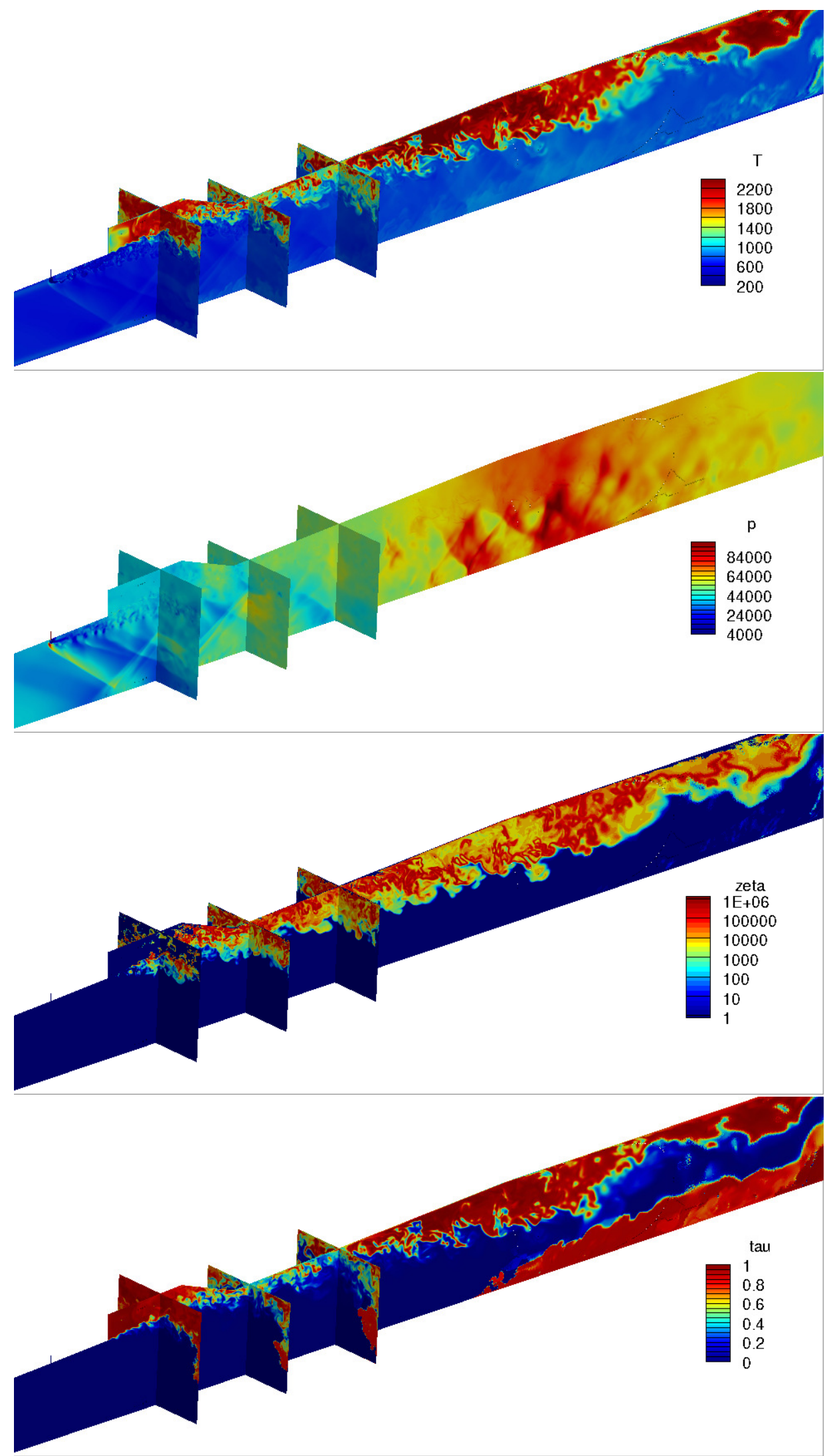

Figure 10. UVa EVM-LES results: instantaneous countours of $T, p, \zeta$, and $\tau$.

\section{6 of 19}



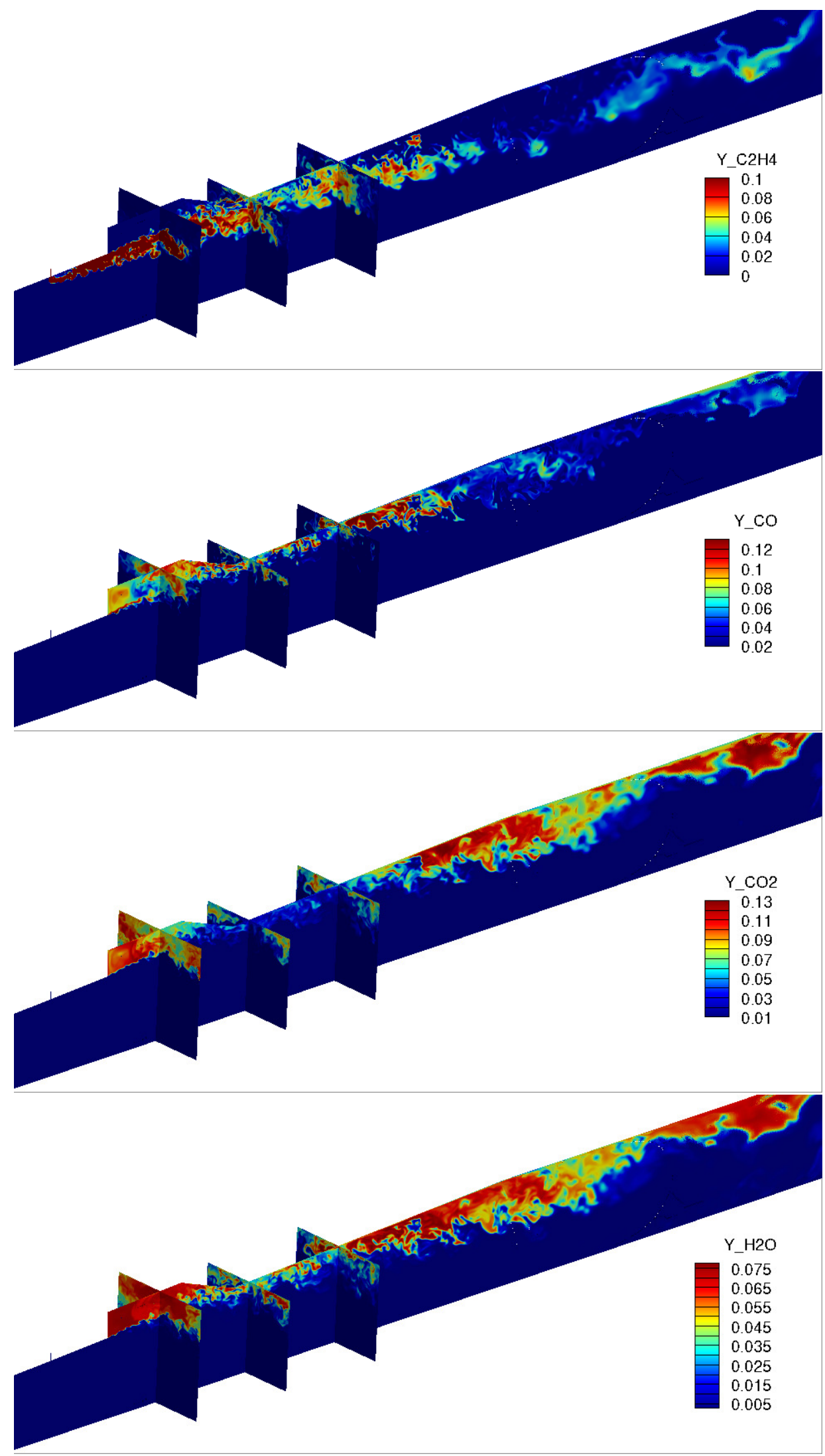

Figure 11. UVa EVM-LES results: instantaneous countours of $\mathrm{C}_{2} \mathrm{H}_{4}, \mathrm{CO}, \mathrm{CO}_{2}$, and $\mathrm{H}_{2} \mathrm{O}$ mass fractions. 


\section{Acknowledgments}

This work was sponsored by the Air Force Office of Scientific Research under grant FA9550-12-1-0461. The views and conclusions contained herein are those of the author and should not be interpreted as necessarily representing the official policies or endorsements, either expressed or implied, of the AFOSR or the U.S. Government.

We would also like to thank Prof. Mirko Gamba of the University of Michigan for providing the code to compute the synthetic OH PLIF signal plots (Fig. 7), Drs. Matthew Bartkowicz and Travis Drayna of GoHypersonic Inc. for generating the grids used in this work, and Mr. Anand Kartha of the University of Minnesota for providing synthetic turbulent inflow code used for the University of Virginia simulations.

\section{References}

${ }^{1}$ Cybalist, N., and P.E. Dimotakis, "On Autoignition-Dominated Supersonic Combustion," AIAA-2015-2315, June 2015.

${ }^{2}$ Pierce, C.D., and P. Moin, "Progress-Variable Approach for Large-Eddy Simulation of Non-Premixed Turbulent Combustion," Journal of Fluid Mechanics, Vol. 204, pp. 73-97, 2004.

${ }^{3}$ Candler, G.V., N. Cymbalist, and P.E. Dimotakis, "Large-Eddy Simulation of Autoignition-Dominated Supersonic Combustion," AIAA-2015-3340, June 2015.

${ }^{4}$ Candler, G.V., H.B. Johnson, I. Nompelis, P.K. Subbareddy, V. Gidzak, and M.D. Barnhardt, "Development of the US3D Code for Advanced Compressible and Reacting Flow Simulations," AIAA SciTech, Jan. 2015.

${ }^{5}$ Burke, M.P., M. Chaos, Y. Ju, F.L. Dryer, and S.J. Klippenstein, "Comprehensive $\mathrm{H}_{2} / \mathrm{O}_{2}$ Kinetic Model for High-Pressure Combustion," International Journal of Chemical Kinetics, Vol. 44, pp. 444-474, 2012.

${ }^{6}$ Gamba, M., and M.G. Mungal, "Ignition, Flame Structure and Near-Wall Burning in Transverse Hydrogen Jets in Supersonic Crossflow," Journal of Fluid Mechanics, Vol. 780, pp. 226-273, 2015

${ }^{7}$ McRae, C.D., C.T. Johansen, P.M. Danehy, Fulton, J., J. Edwards, H. Hassan, R. Rockwell, C. Goyne, J. McDaniel, C. Smith, A. Cutler, C. Johansen, P. Danehy, and T. Kouichi, "Large-Eddy/Reynolds-averaged Navier-Stokes Simulation of a Dual-Mode Scramjet Combustor," AIAA-2012-0115, Jan. 2012. E.C.A. Gallo, L.M.L. Cantu, G. Magnotti, A. Cutler, R. Rockwell, C. Goyne, and J. McDaniel, "OH PLIF Visualization of the UVa Combustion Experiment: Configuration C," AIAA 2013-0034, Jan. 2013.

${ }^{8}$ Potturi, A.S., and J.R. Edwards, "Large-Eddy/Reynolds-Averaged Navier-Stokes Simulation of Cavity-Stabilized Ethylene Combustion," Combustion and Flame, Vol. 162, pp. 1176-1192, 2015.

${ }^{9}$ Chan, W.L., and M. Ihme, "Large-Eddy Simulations of Dual-Mode Scramjet Combustor: Operating Point "A" of University of Virginia's Scramjet Experiments," AIAA-2014-1161, Jan. 2014.

${ }^{10}$ Ben-Yakar, A., M.G. Mungal, and R.K. Hanson, "Time Evolution and Mixing Characteristics of Hydrogen and Ethylene Transverse Jets in Supersonic Crossflows," Physics of Fluids, Vol. 18, 026101, 2006.

${ }^{11}$ UCSD, 2012. Chemical-kinetic mechanisms for combustion applications. San Diego Mechanism web page, Mechanical and Aerospace Engineering (Combustion Research); (http://combustion.ucsd.edu).

${ }^{12}$ Saghafian, A., V.E. Terrapon, and H. Pitsch, "An Efficient Flamelet-Based Combustion Model for Compressible Flows," Combustion and Flame, Vol. 162, Issue 3, pp. 652-667, March 2015.

${ }^{13}$ Oevermann, M., "Numerical Investigation of Turbulent Hydrogen Combustion in a SCRAMJET using Flamelet Modeling," Aerospace Science and Technology, Vol. 4, pp. 463-480, 2000.

${ }^{14}$ McBride, B.J., and S. Gordon, "Computer Program for Calculation of Complex Chemical Equilibrium Compositions and Applications," NASA RP-1311, June 1996.

${ }^{15}$ Quinlan, J.R., T.G. Drozda, J.C. McDaniel, G. Lacaze, and J. Oefelein, "A Priori Analysis of a Compressible Flamelet Model using RANS Data for a Dual-Mode Scramjet Combustor," AIAA Paper 2015-3208, June 2015.

${ }^{16}$ Candler, G.V., P.K. Subbareddy, and J.M. Brock, "Advances in Computational Fluid Dynamics Methods for Hypersonic Flows," J. Spacecraft and Rockets, Vol. 52, No. 1, pp. 17-28, Jan.-Feb. 2015.

${ }^{17}$ MacCormack, R.W., Numerical Computation of Compressible and Viscous Flow, AIAA Education Series, 2014.

${ }^{18}$ Roe, P., "Characteristic-Based Schemes for the Euler Equations," Annual Review of Fluid Mechanics, Vol. 18, pp. 337$365,1986$.

${ }^{19}$ van Leer, B., "Towards the Ultimate Conservative Difference Scheme, V. A Second Order Sequel to Godunov's Method," J. Computational Physics, Vol. 32, pp. 101-136, 1979.

${ }^{20}$ Subbareddy, P., and G.V. Candler, "A Fully-Discrete, Kinetic Energy Consistent Finite Volume Scheme for Compressible Flows," J. Computational Physics, Vol. 228, pp. 1347-1364, Mar. 2009.

${ }^{21}$ Ducros, F., V. Ferrand, F. Nicoud, C. Weber, D. Darracq, C. Gacherieu, T. Poinsot, "Large-Eddy Simulation of Shock/Turbulence Interaction," J. Computational Physics, Vol. 152, No. 2 pp. 517-549, 1999.

${ }^{22}$ Wright, M.J., D. Bose, and G.V. Candler, "A Data-Parallel Line Relaxation Method for the Navier-Stokes Equations," AIAA Journal, Vol. 36, No. 9, pp. 1603-1609, Sept. 1998.

${ }^{23}$ Wright, M.J., G.V. Candler, and M. Prampolini, "Data Parallel Lower-Upper Relaxation Method for the Navier-Stokes Equations," AIAA Journal, Vol. 34, No. 7, pp. 1371-1377, July 1996.

${ }^{24}$ LINK3D V.0.9.0, T.W. Drayna, C.J.W. Haag, M.D. Bartkowicz, and V.M. Gidzak, GoHypersonic Inc., Minneapolis MN, 2016.

${ }^{25}$ Spalart, P.R., and Allmaras, S.R., "A One-Equation Turbulence Model for Aerodynamic Flows," AIAA Paper 92-0439, Jan. 1992. 
${ }^{26}$ Catris, S., and B. Aupoix, "Density Corrections for Turbulence Models," Aerospace Science and Technology, Vol. 4, 2000, pp. 1-11.

${ }^{27}$ Shur, M. L., P.R. Spalart, M.K. Strelets, and A.K. Travin, "A Hybrid RANS-LES Approach with Delayed-DES and Wall-Modelled LES Capabilities," Int. J. Heat and Fluid Flow, Vol 29, No. 6, pp. 16381649, 2008.

${ }^{28}$ Peterson, D.M., G.V. Candler, "Hybrid Reynolds-Averaged and Large-Eddy Simulation of Normal Injection into a Supersonic Crossflow," J. Propulsion and Power, Vol. 26, No. 3, pp. 533-544, 2010.

${ }^{29}$ Gamba, M., "Volumetric PIV and OH PLIF Imaging in the Far Field of Nonpremixed Jet Flames," Ph.D. Thesis, University of Texas, 2009.

${ }^{30}$ Klein, M., A. Sadiki, J. Janicka, "A Digital Filter Based Generation of Inflow Data for Spatially Developing Direct Numerical or Large Eddy simulations," Journal of computational Physics, Vol. 186, No. 2, pp. 652-665, 2003.

${ }^{31}$ Xie, Z.-T. and I.P. Castro, "Efficient Generation of Inflow conditions for Large Eddy Simulation of Street-Scale Flows," Flow, Turbulence and Combustion, Vol. 81, No. 3, pp. 449-470, 2008.

${ }^{32}$ Touber, E., and N.D. Sandham, "Large-Eddy Simulation of Low-Frequency Unsteadiness in a Turbulent Shock-Induced Separation Bubble," Theoretical and Computational Fluid Dynamics, Vo. 23, No. 2, pp. 79-107, 2009. 